\title{
Experimental characterization and numerical modeling of the three-dimensional anisotropic behavior of a thick sheet aluminum alloy AA2024-T351 using multi-scale approaches
}

\author{
W. Nasri ${ }^{1,2}$, A. Gavrus ${ }^{1}$, A. Kouadri-David ${ }^{1} \&$ K. Sai ${ }^{2}$ \\ ${ }^{1}$ LGCGM, INSA Rennes, France \\ ${ }^{2} L G P M M$, ENI Sfax, Tunisia
}

\begin{abstract}
The purpose of this work is to evaluate the anisotropic behaviour of an AA2024T351 thick plate using three different multi-scales approaches: a macroscopic unified model (based on HILL 48 yield loci), a multi-mechanisms model and a polycrystalline one. The experimental results obtained from uniaxial tensile tests are first described. Thereafter, theoretical formulations and identification of studied numerical elastoplastic models are presented. Finally, finite element simulations of tensile tests and analysis of drawing process are performed.

Keywords: thick sheet anisotropy, multi-scale modelling, numerical simulation, aluminium alloy.
\end{abstract}

\section{Introduction}

Recently, different industries faced the challenge of implementation of aluminium alloys in order to produce components with different formability characteristics. In aeronautical manufacturing, due to the need for low-weight aircraft, aluminium alloy elements are used characterized by good mechanical resistance and formability. Generally this material is obtained in sheet form by hot and cold rolling forming processes which creates plastic anisotropy. It is then important to understand the anisotropic mechanical behavior characterising a thin [1] or thick sheet and ensure the reliability of the numerical models predictions in order to be integrated in an optimal design analysis [2]. This work presents an introduction of the study of the three-dimensional anisotropy of an aluminium alloy. So the paper starts with two specific cases of a 3D plastic anisotropy: plane and normal, 
analyzing three different elasto-plastic numerical approaches: a unified model, a multi-mechanism model and a polycrystalline one [3-10]. To demonstrate their feasibility and to identify the most accurate model describing the plastic anisotropy, a lot of comparisons are realized with the experimental data obtained from tensile tests performed for specimens with a thickness of $3 \mathrm{~mm}$ along different directions to the rolling direction. Using the three different approaches numerical finite element (FE) analyses of tensile tests and drawing process simulations are presented.

\section{Experimental tensile tests}

Uniaxial tensile tests were performed for an AA2024-T351 aluminium alloy plate at different directions: $0^{\circ}, 30^{\circ}, 45^{\circ}, 60^{\circ}, 75^{\circ}$ and $90^{\circ}$ with respect to the initial rolling direction $\left(0^{\circ}\right)$, using an INSTRON machine $(50 \mathrm{KN}$ load cell), recording both the loads and displacements for an initial strain rate of $2 \times 10^{-3} \mathrm{~s}^{-1}$.

\subsection{The sample material}

In this work, all specimens were cutting out from the thick aluminium alloy rolling plate (thickness of $3 \mathrm{~mm}$ ).

Figure 1 shows the used specimen's geometry, the corresponding dimensions and figure 2 pictured the specimen mounting.

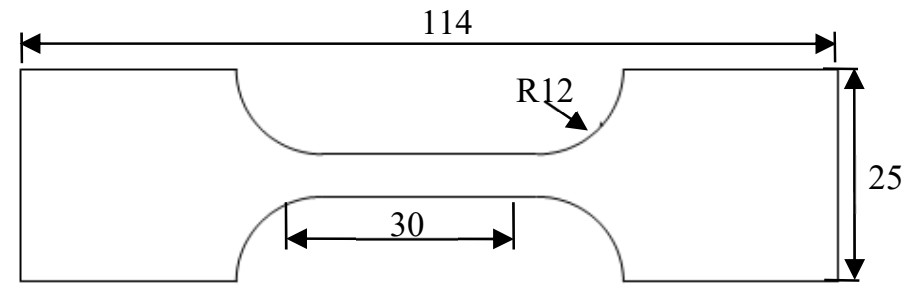

Figure 1: $\quad$ Plane specimen dimensions (in $\mathrm{mm}$ ).
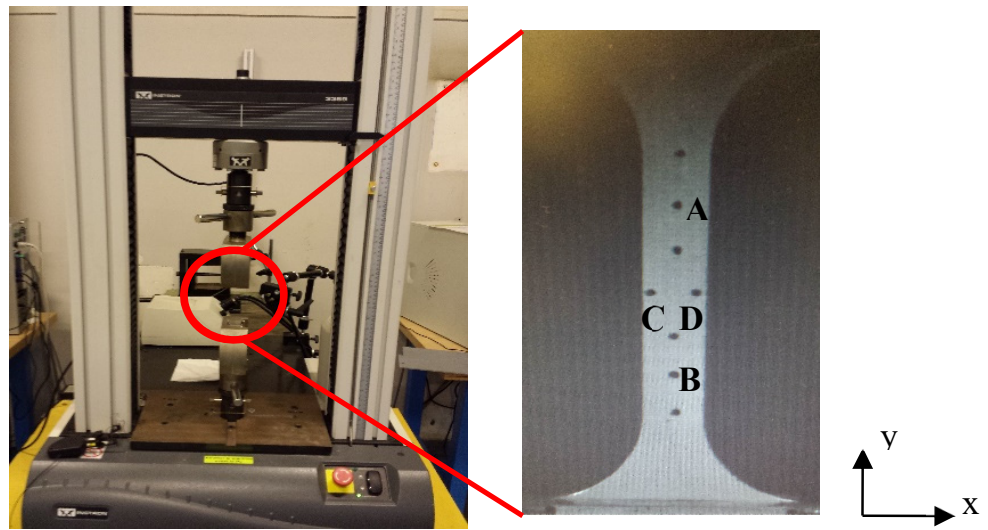

Figure 2: INSTRON universal testing machine and the tasks points (A, B, C, D) positioned on the tensile specimen surface. 


\subsection{Experimental results and classical analysis}

Axial load and displacements in the longitudinal and transverse directions were recorded during the experiment using a speed camera measuring point's displacements throughout the material traction (figure 2). In fact, the camera followed two points $\mathrm{A}$ and $\mathrm{B}$ along the $\mathrm{y}$ axis of loading (having an initial distance $1_{0}$ between them) and two points $\mathrm{C}$ and $\mathrm{D}$ along the $\mathrm{x}$ axis (spaced by an initial distance $b_{0}$ ). From digital images of the specimen is performed evolution of each local distance and computed the corresponding total strains $\varepsilon_{y y}^{t}, \varepsilon_{x x}^{t}$. The plastic strain is obtained by assuming the partition of the total strain of an elastic part and a plastic one i.e. $\varepsilon^{t}=\varepsilon^{e}+\varepsilon^{p}$, giving: $\varepsilon^{p}=\varepsilon^{t}-\varepsilon^{e}$. The plastic strain according to the three orthotropic axes $(x, y, z)$ can then be calculated as follows: $\varepsilon_{y y}^{p}=\varepsilon_{y y}^{t}-\sigma_{y y} / E, \varepsilon_{x x}^{p}=\varepsilon_{x x}^{t}+v \sigma_{y y} / E$ and $\varepsilon_{z z}^{p}=-\left(\varepsilon_{x x}^{p}+\varepsilon_{y y}^{p}\right)$ if assumption of plastic incompressibility. Then both stress-strain curves and Lankford coefficients (defined by $r=\frac{\dot{\varepsilon}_{x x}^{p}}{\dot{\varepsilon}_{z z}^{p}}=\frac{d \varepsilon_{x x}^{p}}{d \varepsilon_{z z}^{p}} \approx \frac{\varepsilon_{x x}^{p}}{\varepsilon_{z z}^{p}}$ ) have been computed from different loadings directions, especially for $0^{\circ}, 45^{\circ}$ and $90^{\circ}$. As can be seen in figure 3 , a similar material behavior is obtained between the tensile directions $45^{\circ}$ (DD) and $90^{\circ}$ (TD).

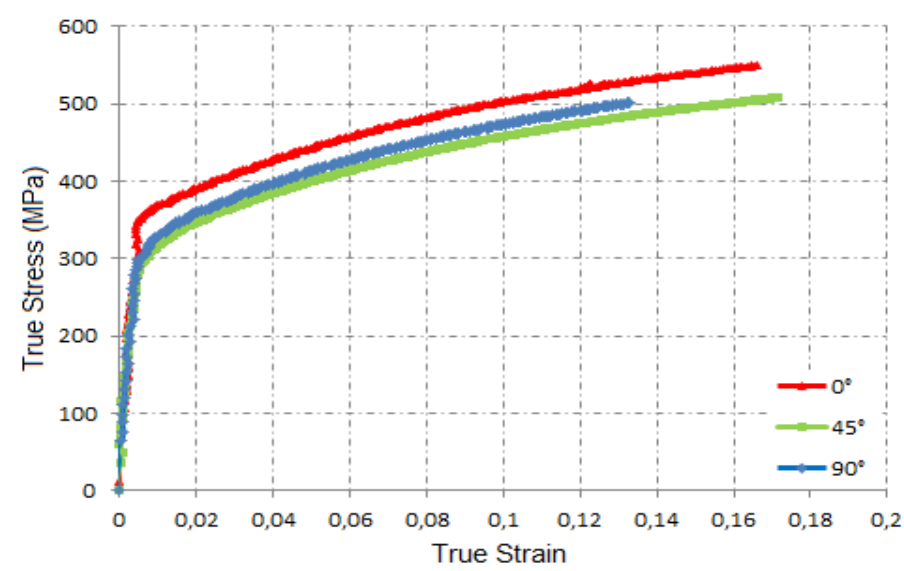

Figure 3: Experimental true stress-true strain curve along the directions $0^{\circ}, 45^{\circ}$ and $90^{\circ}$ of the AA2024-T351 thick plate (present work).

The plastic behavior of the specimen loaded at $0^{\circ}$ (RD) is slightly different in intensity with a dozen of MPa. Regarding the figure 4, some differences can be observed as compared to thin sheets [1]. Concerning the elastic yield stress $\sigma 00$ this one has average value about $300 \mathrm{MPa}$ (between $250 \mathrm{MPa}$ and $325 \mathrm{MPa}$ ), slightly higher than that of the thin sheet $(250 \mathrm{MPa})$. 
166 High Performance and Optimum Design of Structures and Materials II

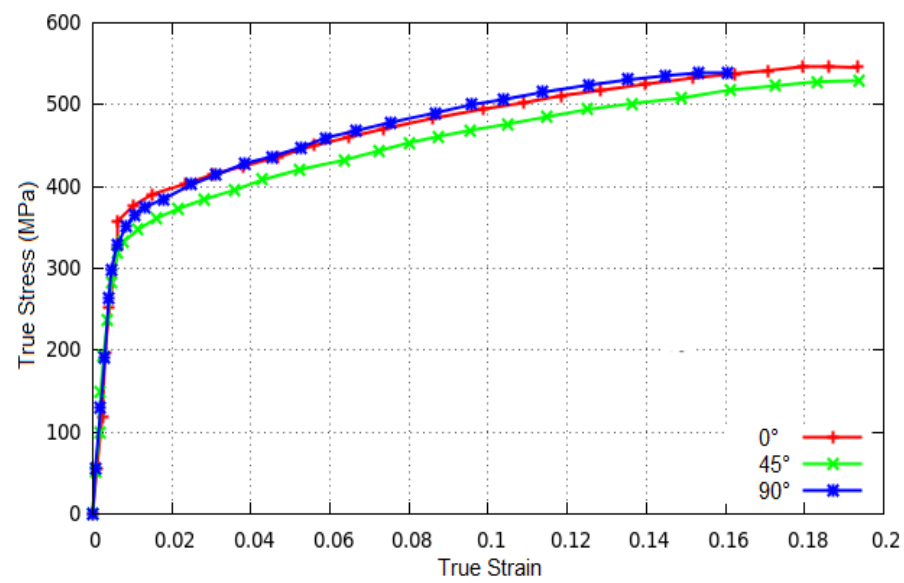

Figure 4: Experimental true stress-true strain curve along the directions $0^{\circ}, 45^{\circ}$ and $90^{\circ}$ of an AA2024-T351 thin sheet [1].

Starting from the classical plane anisotropy theory (used for studying thin sheets) the material anisotropy can be firstly estimated via the Lankford coefficient $r(\theta)$. The values measured for different angles and at various plastic strain levels are listed in table 1. Figure 5 plot the corresponding variations. Because the planar anisotropy $\Delta r$ has smaller values ( $\Delta r=-0.05$ for $5 \%$ plastic deformation and $\Delta r$ $=-0.07$ for $10 \%$ ) it is possible to consider that this aluminum alloy has rather a normal anisotropy, which is a particular case of a 3D anisotropy.

Table 1: The $r(\theta)$ values corresponding to different tensile directions (angle $\theta$ ) and corresponding to different plastic strain levels.

\begin{tabular}{|c|c|c|c|c|c|}
\hline \multicolumn{5}{|c|}{$\mathrm{r}(\theta)$} \\
\hline $\begin{array}{c}\text { Cumulated } \\
\text { plastic strain }\end{array}$ & $2 \%$ & $5 \%$ & $7 \%$ & $10 \%$ & $12 \%$ \\
\hline $0^{\circ}$ & 0.88 & 0.75 & 0.61 & 0.62 & 0.61 \\
\hline $30^{\circ}$ & 0.74 & 0.59 & 0.56 & 0.79 & 0.71 \\
\hline $45^{\circ}$ & 0.6 & 0.78 & 0.7 & 0.68 & 0.73 \\
\hline $60^{\circ}$ & 0.6 & 0.53 & 0.57 & 0.78 & 0.68 \\
\hline $75^{\circ}$ & 1.19 & 0.78 & 1.03 & 0.7 & 0.61 \\
\hline $90^{\circ}$ & 0.83 & 0.7 & 0.67 & 0.6 & 0.59 \\
\hline$\overline{\mathrm{r}}$ & 0.73 & 0.75 & 0.67 & 0.64 & 0.66 \\
\hline$\Delta \mathrm{r}$ & 0.25 & -0.05 & -0.06 & -0.07 & -0.13 \\
\hline
\end{tabular}




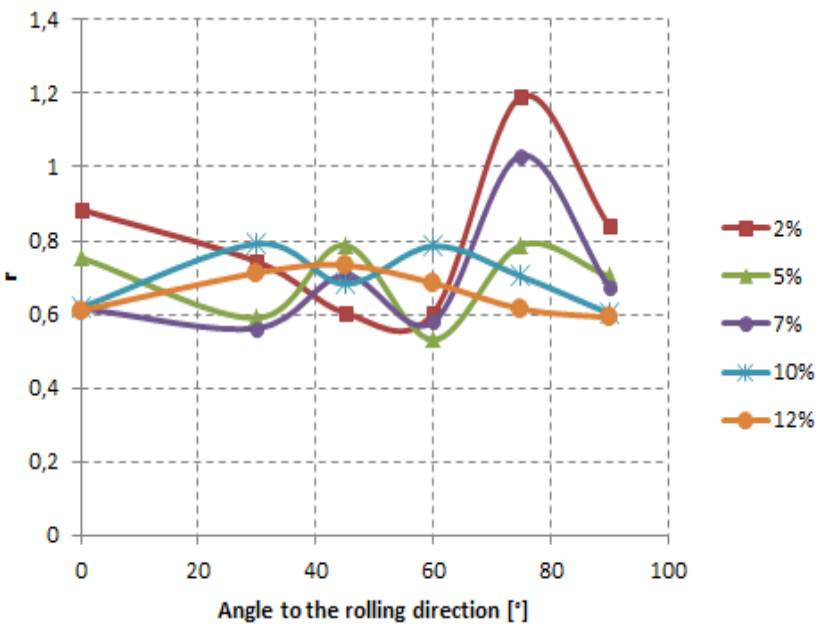

Figure 5: Variation of $r(\theta)$ with the equivalent plastic strain.

Moreover, an average Lankford coefficient $\bar{r}$ lower than $1(\bar{r}=0.75$ for $5 \%$ plastic deformation and $\bar{r}=0.64$ for $10 \%$ ) reflects a less good formability of this material. Starting from a Hill anisotropic law, the variations of the coefficients with the plastic strain $\left(F^{\prime}, G^{\prime}, H^{\prime}, L^{\prime}, M^{\prime}, N^{\prime}\right.$ or dimensionless $F, G, H, L, M, N$ defined by the relationships of Table 2) seems to be in accordance with the classical analysis of the plane and normal anisotropy formulation. The evolution of these coefficients with the plastic strain is plotted in figures 6 . In a first approximation, estimating $\mathrm{r}$ coefficients for $0.2 \%$ of plastic deformation, the dimensionless Hill coefficients $F, G, H$ and $N$ have constant values. In the case of normal anisotropy, in order to describe in a more realistic way the variation of the

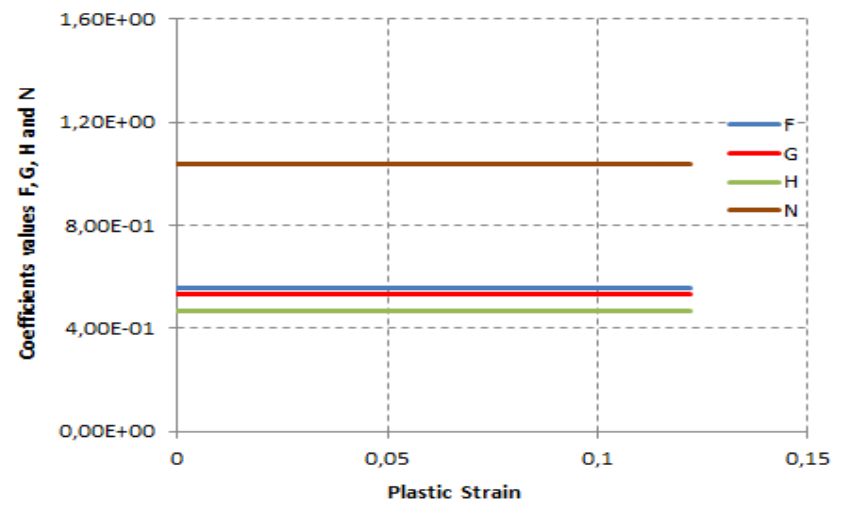

Figure 6: Variations of the computed Hill coefficients as a function of the plastic deformation for a plane anisotropy. 
average Lankford coefficient $\bar{r}$, one makes a three degree polynomial interpolation (Figure 7). The corresponding curves representing the variation of the computed Hill coefficients are plotted in figure 8 .

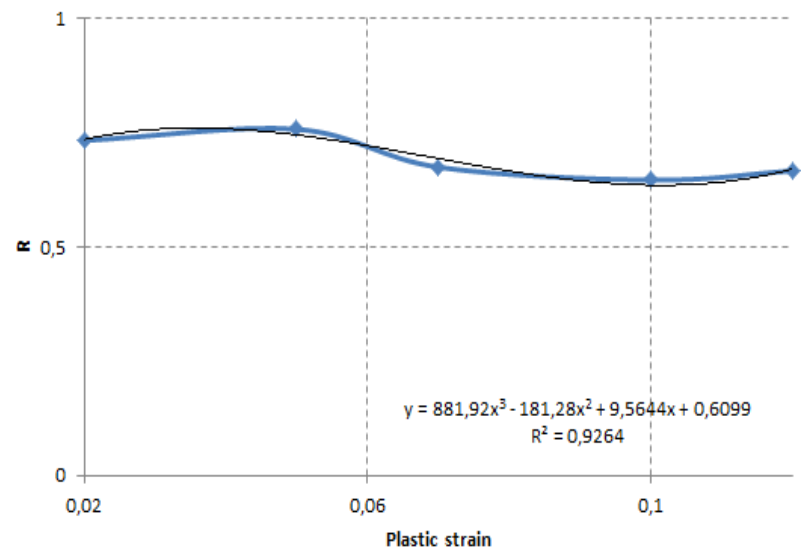

Figure 7: Three degree polynomial interpolation of $\bar{r}$ according to the variation with the plastic strain.

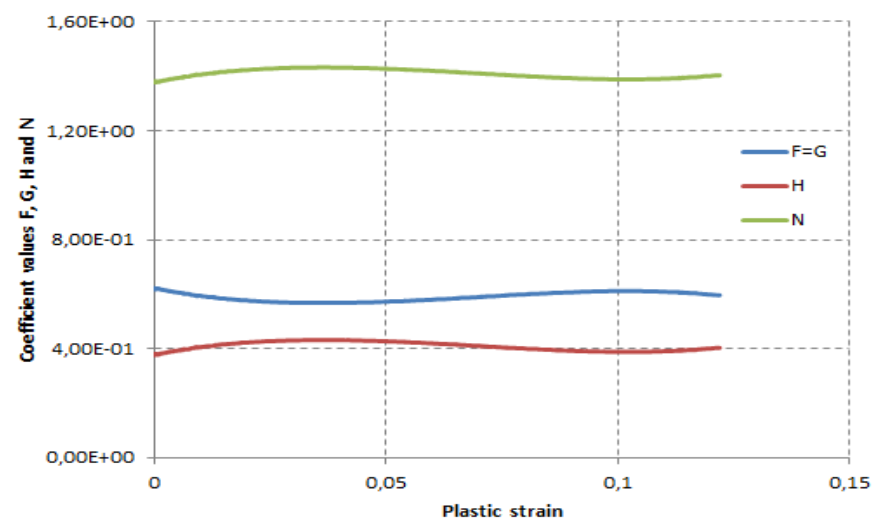

Figure 8: Variations of the computed Hill coefficients as a function of the plastic deformation for a normal anisotropy.

\section{Constitutive models}

The use of a finite strain formulation through updated Lagrangian formalism is needed to model large-strain deformations. The material behavior description is based on constitutive equations using internal tensorial variables [2] and on the Green-Naghdi transformation of the stress-strain problem. The strain is decomposed into elastic part and inelastic parts i.e. $\tilde{\varepsilon}=\tilde{\varepsilon}^{e}+\tilde{\varepsilon}^{p}$ starting from $d \tilde{\varepsilon}=d \tilde{\varepsilon}^{e}+d \tilde{\varepsilon}^{p}$ and $\tilde{\sigma}=\tilde{\tilde{\Lambda}}: \tilde{\varepsilon}^{e}$, where $\tilde{\varepsilon}^{e}$ is the elastic strain calculated from the 
generalized Hook law, $\tilde{\sigma}$ is the Cauchy stress tensor and $\tilde{\tilde{\Lambda}}$ is the fourth-rank tensor of elastic moduli.

\subsection{Macroscopic unified model: Hill ' 48 yield criterion}

There are many possibilities for extending the isotropic criteria to the description of anisotropic materials [3-5]. The method generally used to take into account a $3 \mathrm{D}$ anisotropy is to involve a tensor of fourth order into the criterion expression [5-9]. An anisotropic yield loci can be written as a quadratic extension of the von Mises formulation as can be seen in Table 2 which summarizes the general constitutive equations of a macroscopic unified model and of anisotropic Hill 1948 criteria.

Table 2: Constitutive equations of the unified model [3-9].

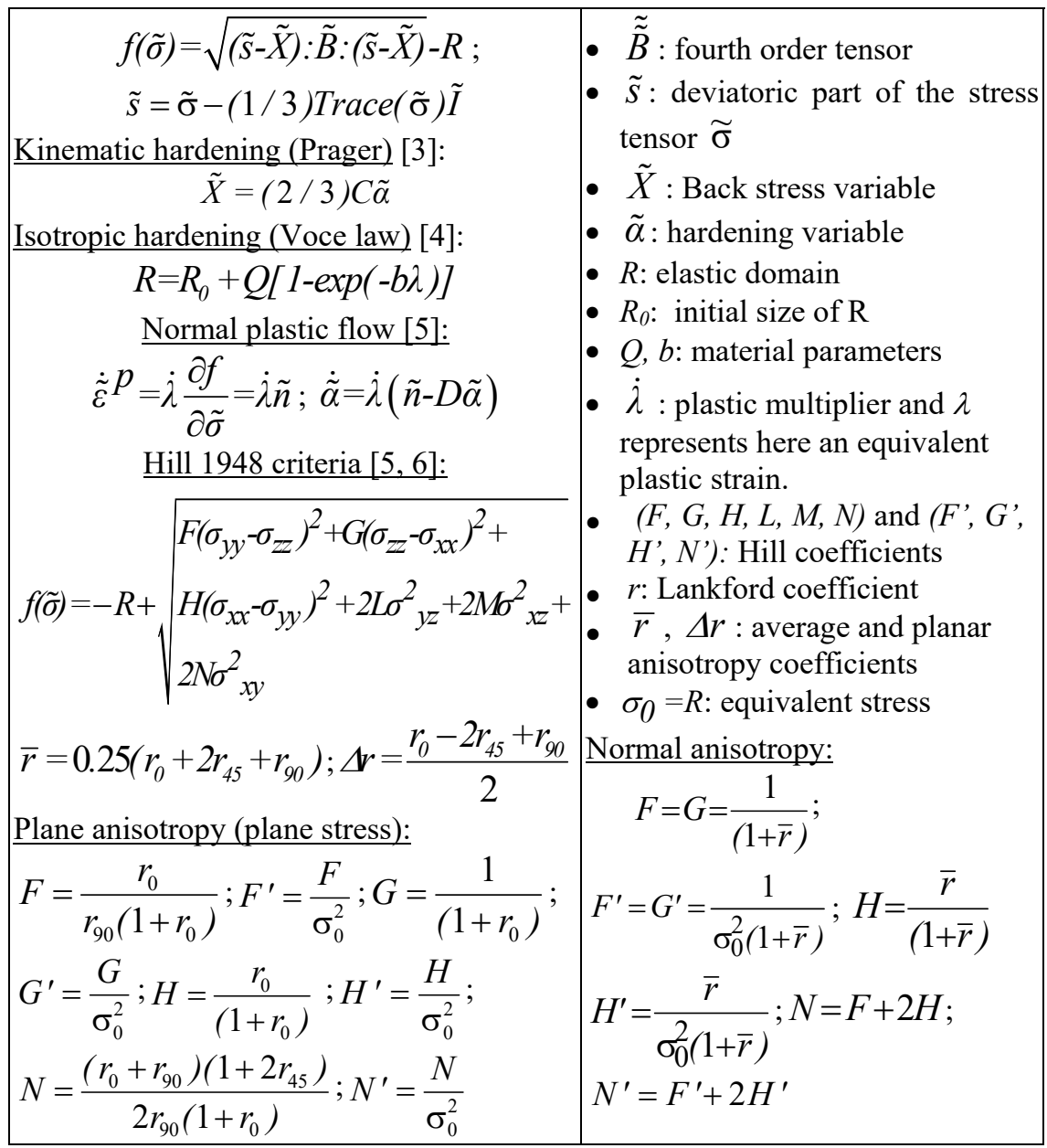




\subsection{Polycrystalline model}

For this type of model, the plastic deformation can be seen as the result of the dislocations slips along the active directions of the crystallographic systems defining the material structure (FCC for AA2024). According to a Cailletaud model [7] Table 3 summarizes the corresponding used equations.

Table 3: Equations of the polycrystalline model [7-9].

\begin{tabular}{|l|l|}
\hline$\tilde{\sigma}^{g}=\tilde{\Sigma}+C\left(\left\langle\tilde{\beta}^{g}\right\rangle-\tilde{\beta}^{g}\right)$ & $\bullet \dot{\tilde{E}}^{p}:$ macro plastic strain rate \\
$\dot{\tilde{\beta}}^{g}=\dot{\tilde{\varepsilon}}^{g}-D \tilde{\beta}^{g}\left\|\dot{\tilde{\varepsilon}}^{g}\right\|, \tau^{s}=\tilde{\sigma}^{g}: \tilde{m}_{s}^{g} ;$ & $\bullet \tilde{\Sigma}:$ macro stress \\
$\tilde{m}_{s}^{g}=\frac{1}{2}\left(\tilde{n}^{s} \otimes l^{s}+l^{s} \otimes \tilde{n}^{s}\right)$ & $\bullet \tilde{\sigma}^{g}:$ local grain stress \\
$\dot{\gamma}_{s}=\left\langle\frac{\left|\tau^{s}-x^{s}\right|-r^{s}}{k}\right\rangle^{n} \operatorname{sig} n\left(\tau^{s}-x^{s}\right)$ & $\bullet \tau^{s}:$ local shear stress \\
$r^{s}=r_{0}^{\prime}+q \sum H^{r s}\left(1-\alpha v^{r}\right) ; \dot{v}^{r}=\left|\dot{\gamma}^{r}\right|$ & $\bullet \tilde{m}_{s}^{g}:$ orientation tensor \\
$\dot{\tilde{\varepsilon}}^{p}=\sum_{s=1}^{12} \tilde{m}_{s}^{g} \dot{\gamma}_{s} ; \dot{\tilde{E}}^{p}=\left\langle\dot{\tilde{\varepsilon}}^{p}\right\rangle$ & $\bullet \dot{\gamma}_{s}:$ shear rate \\
& $\bullet r^{s}:$ isotropic hardening \\
& $\bullet x^{s}:$ kinematic hardening \\
\hline
\end{tabular}

\subsection{Multi-mechanisms model}

The objective of the multi-mechanism models is to use an approach that falls between a macroscopic scale describing a global plastic deformation description and a microscopic one based on crystallographic mechanisms of deformation. The anisotropic behavior of AA2024 alloy is investigated here using a $2 \mathrm{M} 1 \mathrm{C}$ model ( 2 mechanisms and 1 criterion) described in Table 4.

The plastic strain $\widetilde{\varepsilon}^{\mathrm{p}}$ is written as the sum of two components $\widetilde{\varepsilon}^{1}$ and $\widetilde{\varepsilon}^{2}$ which represent the local inelastic strains of the mechanism 1 and 2 respectively. The material anisotropy criterion $f$ (Table 4 ) will be here represented by two second invariants $\mathrm{J}_{\mathrm{L}}$, introducing a fourth order tensor $\tilde{\tilde{\mathrm{L}}}$ where only the non-diagonal terms are different from zero. Then the anisotropic parameters are denoted by the diagonal terms $\mathrm{L}_{\mathrm{a}}, \mathrm{L}_{\mathrm{b}}, \mathrm{L}_{\mathrm{c}}, \mathrm{L}_{\mathrm{d}}, \mathrm{L}_{\mathrm{e}}$ and $\mathrm{L}_{\mathrm{f}}$ of corresponding $\mathrm{L}$ matrix.

\section{Numerical approach}

The numerical implementation of the above three elastoplastic models is performed via the subroutine ZSeT and the material library ZMaT of the FE code ZeBuLoN. In this section, the reliability of the models is evaluated using the results of uniaxial tensile tests according to the three principal directions $0^{\circ}(\mathrm{RD})$, $45^{\circ}$ (DD) and $90^{\circ}$ (TD). For all these simulations are used an isotropic hardening Voce law and a Prager's equation if kinematic hardening (see Table 2). It should 
Table 4: Multi-mechanisms models (MM-2M1C) [8-10].

\begin{tabular}{|c|c|}
\hline $\begin{array}{l}\tilde{\varepsilon}^{p}=\tilde{\varepsilon}^{1}+\tilde{\varepsilon}^{2} \\
f=\sqrt{\left(J_{L}\left(\tilde{\sigma}-\tilde{X}^{I}\right)\right)^{2}+\left(J_{L}\left(\tilde{\sigma}-\tilde{X}^{2}\right)\right)^{2}}-R^{\prime} \\
J_{L I}=J_{L}\left(\tilde{\sigma}-\tilde{X}^{I}\right)=\sqrt{\frac{3}{2}\left(s-\tilde{X}^{I}\right): \tilde{\tilde{L}}:\left(\tilde{s}-\tilde{X}^{I}\right)} \\
I=1,2 \operatorname{and} R^{\prime}=R_{0}^{\prime}+Q^{\prime}\left[1-\exp \left(-b^{\prime} \lambda\right)\right] \\
\left(\begin{array}{c}\tilde{X}^{1} \\
\tilde{X}^{2}\end{array}\right)=\frac{2}{3}\left(\begin{array}{ll}C_{11} & C_{12} \\
C_{12} & C_{11}\end{array}\right)\left(\begin{array}{c}\tilde{\alpha}^{1} \\
\tilde{\alpha}^{2}\end{array}\right) \\
\tilde{n}^{I}=\frac{3}{2} \frac{\tilde{\tilde{L}}:\left(\tilde{s}-\tilde{X}^{I}\right)}{\left(J_{L I}^{2}+J_{L I}^{2}\right)^{I / 2}}\end{array}$ & $\begin{array}{l}\text { - } f: \text { yield criterion function } \\
\text { - } \tilde{\varepsilon}^{p}: \text { plastic strain } \\
\text { - } \tilde{\varepsilon}^{I}: \text { local inelastic strains of the } \\
\text { mechanism I }(1,2) \\
\text { - } \tilde{X}^{I}: \text { back stress of each } \\
\text { mechanism I } \\
\text { - } C_{11}, C_{12}, C_{22}: \text { kinematic } \\
\text { hardening moduli } \\
\text { - } \tilde{n}^{I}: \text { normal to the yield surface } \\
\text { for each mechanism } \\
\text { - } \tilde{\alpha}^{I}: \text { kinematic internal variables }\end{array}$ \\
\hline
\end{tabular}

be mentioned that for each model the identification of material parameters were carried in two stages. In a first time by changing their values via an interactivegraphic method until the computed FE curves and the experimental ones have close variations. At this stage, an optimization module of $\mathrm{ZeBuLoN}$ is started to improve the solution by an automatic non-linear regression. Figure 9 shows simulation results of a tensile test using the unified model with a Voce law (without kinematic hardening) defined by the following variation of the equivalent stress: $R=301+250[1-\exp (-18 \lambda)]$.

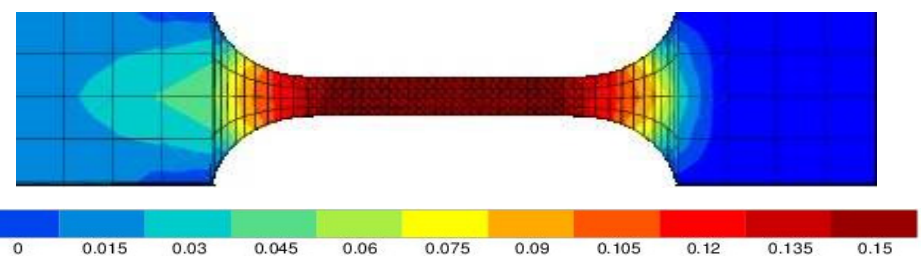

Figure 9: Evolution of plastic deformation obtained by FE simulation of the tensile test along the $0^{\circ}$ direction.

It can be observed the homogeneous strain state of the useful specimen part, validating then the experimental estimations of the stress and plastic strain.

\subsection{Macroscopic unified model}

FE simulations of tensile tests are used to identify the anisotropic parameters (Table 5).

According to $G+H=1$ only three independent anisotropic parameters $\mathrm{F}, \mathrm{G}$ and $N$ must to be identified. Anisotropic parameters $\mathrm{L}$ and $\mathrm{M}$ cannot be determined considering only uniaxial tensile tests. It is therefore assumed that $L=M=N$. It is 
important to mention that identification was realized in the case with a kinematic hardening, but outside the Hill parameters values, the material coefficients have not a unique solution. In this case it should be introduced other experimental data as those obtained from cyclic tests. In figure 10, the stress-strain curves show that there is a good agreement between the experiment and simulation.

Table 5: Identified parameters of the anisotropic unified model without kinematic hardening (with kinematic hardening) (MPa).

\begin{tabular}{|c|c|c|c|c|c|c|c|}
\hline \multicolumn{2}{|c|}{ Elasticity } & \multicolumn{3}{c|}{ Isotropic hardening } & \multicolumn{2}{c|}{ Kinematic hardening } \\
\hline $\mathrm{E}$ & $\mathrm{v}$ & $\mathrm{R}_{0} \approx \sigma_{00}$ & $\mathrm{Q}$ & $\mathrm{b}$ & $\mathrm{C}$ & \multicolumn{2}{c|}{$\mathrm{D}$} \\
\hline 80000 & 0.33 & 301 & 250 & 18 & 0 & \multicolumn{2}{c|}{0} \\
$(80000)$ & $(0.33)$ & $(301)$ & $(260)$ & $(15)$ & $(17000)$ & $(2200)$ \\
\hline \multicolumn{7}{|c|}{ Plane anisotropy } \\
\hline $\mathrm{F}$ & $\mathrm{G}$ & $\mathrm{H}$ & $\mathrm{L}$ & $\mathrm{M}$ & $\mathrm{N}$ \\
\hline 0.5 & 0.63 & 0.37 & 0.97 & 0.97 & 0.97 \\
$(0.41)$ & $(0.62)$ & $(0.38)$ & $(1.25)$ & $(1.25)$ & $(1.25)$ \\
\hline \multicolumn{6}{|c|}{ Normal anisotropy } \\
\hline $\mathrm{F}$ & $\mathrm{G}$ & $\mathrm{H}$ & $\mathrm{L}$ & $\mathrm{M}$ & $\mathrm{N}$ \\
\hline 0.63 & 0.63 & 0.36 & 1.35 & 1.35 & 1.35 \\
$(0.57)$ & $(0.57)$ & $(0.42)$ & $(1.42)$ & $(1.42)$ & $(1.42)$ \\
\hline
\end{tabular}

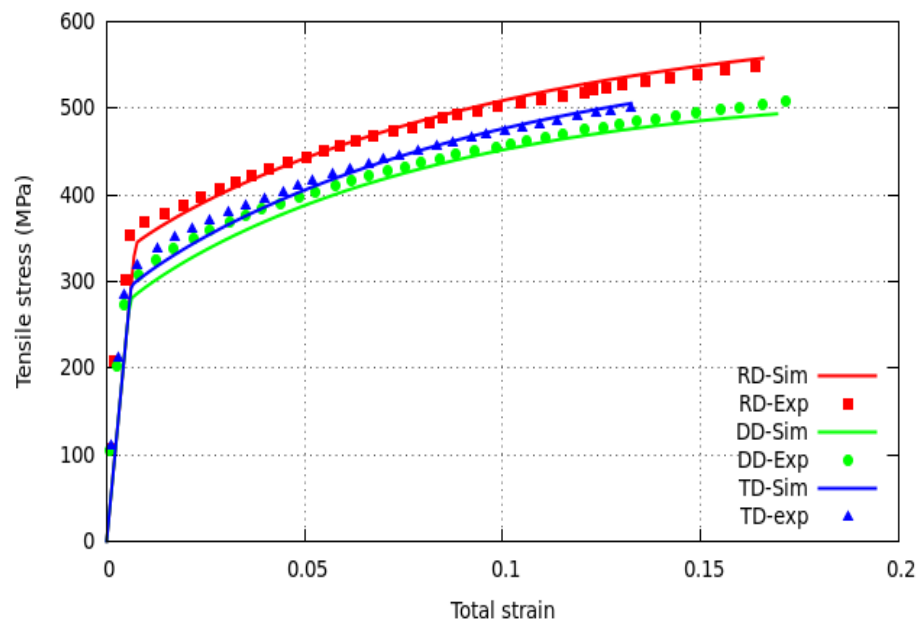

Figure 10: Comparisons between experimental and simulated tensile tests for the unified model without kinematic hardening.

\subsection{Polycrystalline model}

The use of polycrystalline models to describe the anisotropic mechanical behavior of AA2024 aluminum alloy requires taking into account their crystallographic texture provided by the spatial distribution of the grains orientations. An initial 
$\{111\}$ pole figure approximated by a set of 212 orientations corresponding to the initial state of the material and constructed using a numerical stereographic projection code (Projstern) is shown in Figure 11. The identified parameters values of the polycrystalline model obtained from the best fit between the experimental responses and those obtained from FE simulations are given in Table 6.

Table 6: Identified parameters of the polycrystalline model (MPa).

\begin{tabular}{|c|c|c|c|c|c|c|c|c|}
\hline \multicolumn{2}{|c|}{ Elasticity } & \multicolumn{4}{|c|}{ Isotropic hardening } & \multicolumn{2}{|c|}{ Norton parameters } & \multicolumn{2}{|c|}{$\begin{array}{c}\text { Kinematic } \\
\text { hardening }\end{array}$} \\
\hline $\mathrm{E}$ & $\mathrm{v}$ & $\mathrm{r}_{0}{ }_{0}$ & $\mathrm{q}$ & $\alpha$ & $\mathrm{k}$ & $\mathrm{n}$ & $\mathrm{C}$ & $\mathrm{D}$ \\
\hline 75000 & 0.33 & 301 & 90 & 2 & 80 & 8 & 300000 & 100 \\
\hline
\end{tabular}

Figure 12 shows that the stress-strain curves obtained by numerical simulations are in a good agreement with the experiment.
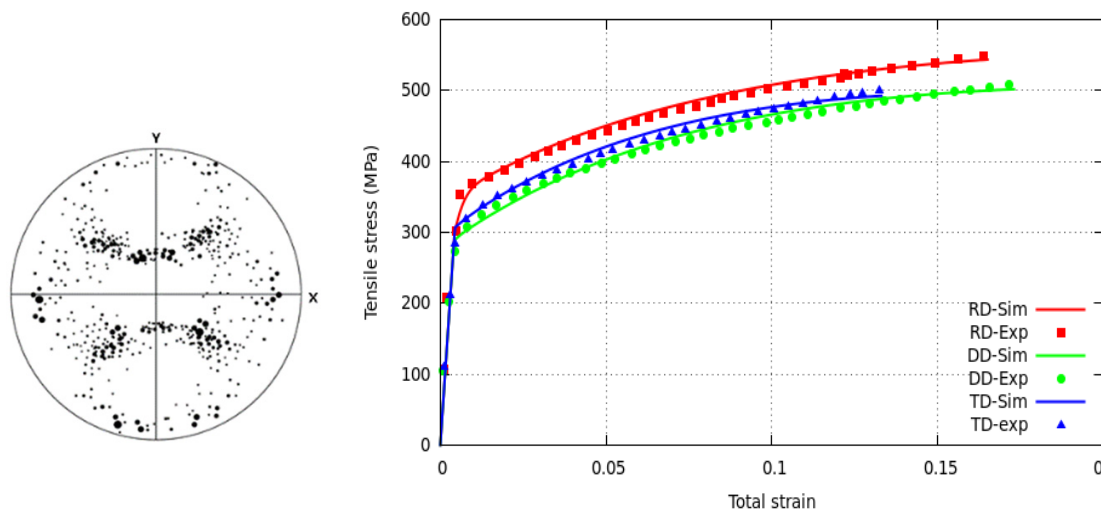

Figure 11: $\{111\} \quad$ Pole Figure 12: Comparisons between experimental fig. computed by Projstern and simulated tensile curves for the [9]. polycrystalline model.

\subsection{Multi-mechanisms model (MM-2M1C)}

The identified anisotropic material parameters are shown in the table 7 (the isotropic parameters Le and Lf are not identified here).

Table 7: Identified parameters of the anisotropic 2M1C model (MPa).

\begin{tabular}{|c|c|c|c|c|c|c|}
\hline $\mathrm{E}$ & $v$ & $\mathrm{R}^{\prime}{ }_{0} \approx \sigma_{00} \sqrt{2}$ & $\mathrm{Q}^{\prime}$ & $\mathrm{C}_{11}$ & $\mathrm{C}_{22}$ & $\mathrm{C}_{12}$ \\
\hline 80000 & 0.33 & 360 & 260 & 1200 & 2500 & 900 \\
\hline $\mathrm{b}^{\prime}$ & $\mathrm{D}_{1}$ & $\mathrm{D}_{2}$ & $\mathrm{~L}_{\mathrm{a}}$ & $\mathrm{L}_{\mathrm{b}}$ & $\mathrm{L}_{\mathrm{c}}$ & $\mathrm{L}_{\mathrm{d}}$ \\
\hline 7 & 5 & 5 & 1 & 1 & 0.2 & 0.5 \\
\hline
\end{tabular}


The comparisons between the simulated stress-strain curves and the experimental ones are illustrated by figure 13 .

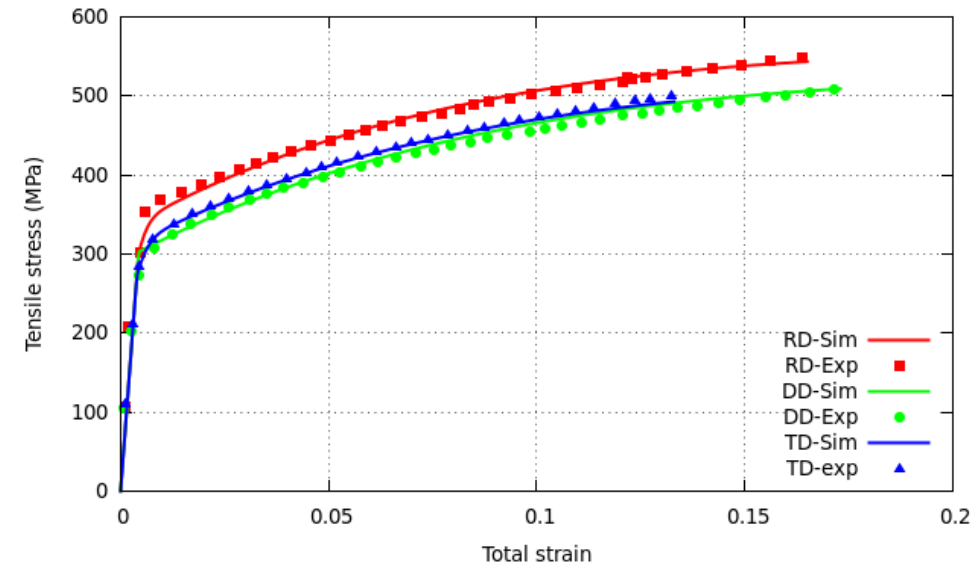

Figure 13: Comparisons between experimental and simulated tensile tests for the MM (2M1C) model.

For this model the computed curves have more precise values as compared to those obtained by the previous models.

\section{Application: thick sheet drawing process}

In this section, a numerical simulation of an axisymmetric (2D) drawing process is performed using FE models. The simple drawing is performed without a blank holder, considering that the sheet is placed on the die and clamped on its edge. The punch and the die are modelled as rigid bodies whereas only the thick blank is defined as a deformable body using a mesh defined by 4-nodes linear elements (CAX4) (figure 14).
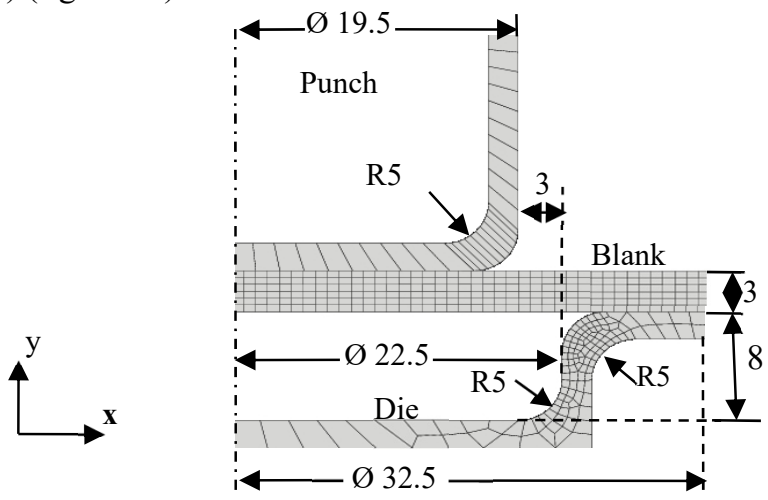

Figure 14: The geometry and the mesh used in a finite element drawing process simulation (all dimensions are in $\mathrm{mm}$ ). 
It is important to mention that this study is focused on the case of a conventional drawing of a thick sheet $(3 \mathrm{~mm})$ using shallow depth $(8 \mathrm{~mm})$. The clearance between the punch and the die is $3 \mathrm{~mm}$ and the friction of contact between punch/blank and blank/die were defined with a Coulomb model via a friction coefficient equal to 0.1 . All the other used material parameters values are listed in table 5 (Unified Model with a plane anisotropy), table 6 (Polycrystalline Model) and table 7 (2M1C Model). Figure 15 shows the numerical load-stroke curves for the three models obtained from simulation with Zebulon code.

For the simulations using an unified model without kinematic hardening both Abaqus and Zebulon code were performed using an isotropic hardening Voce law defined by $R=301+250[1-\exp (-18 \lambda)]$. Figure 16 shows the comparisons of the

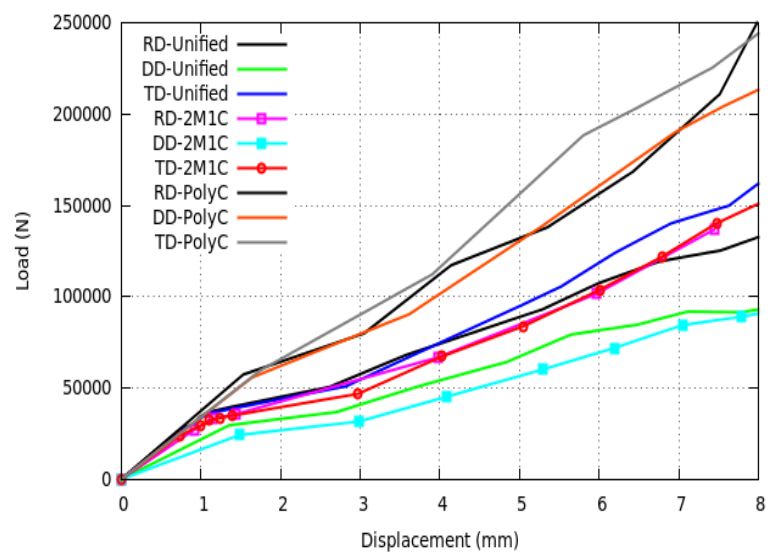

Figure 15: Numerical load-displacement curves obtained for RD, TD and DD drawing tests and the three models: Unified, MM (2M1C) and Polycrystalline (Zebulon).

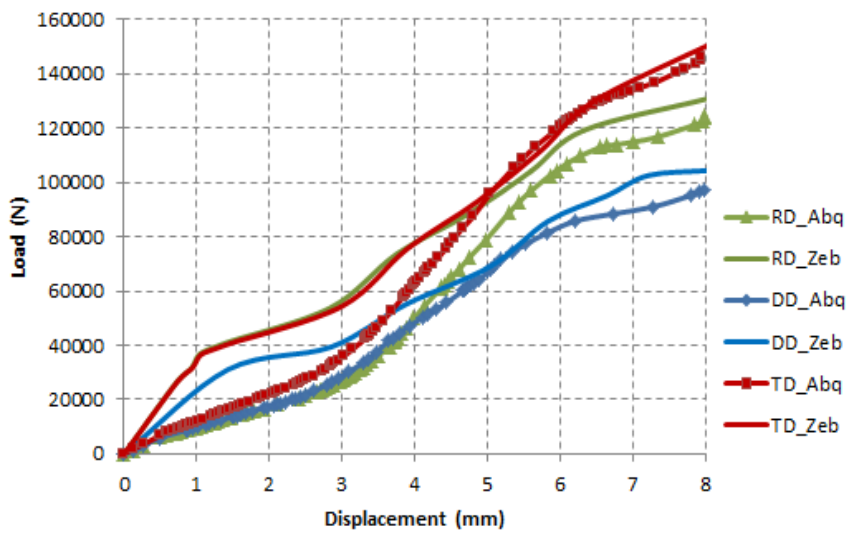

Figure 16: Numerical load-displacement curves obtained for RD, TD and DD drawing tests using the unified model without kinematic hardening (Abaqus and Zebulon). 
numerical load-displacement curves. Nearly similar shape and maximum loads are obtained for unified and MM (2M1C) models as compared to the polycrystalline one which seems to overestimate the drawing forces.

Contour plots of the equivalent stress at the end stage of the loading are shown in figure 17 for the three directions RD, DD and TD. It can be observed that the unified and the MM (2M1C) models lead to similar distribution of the stress. On the other side, the polycrystalline model gives different results principally due to the important influence of the local material texture evolution.

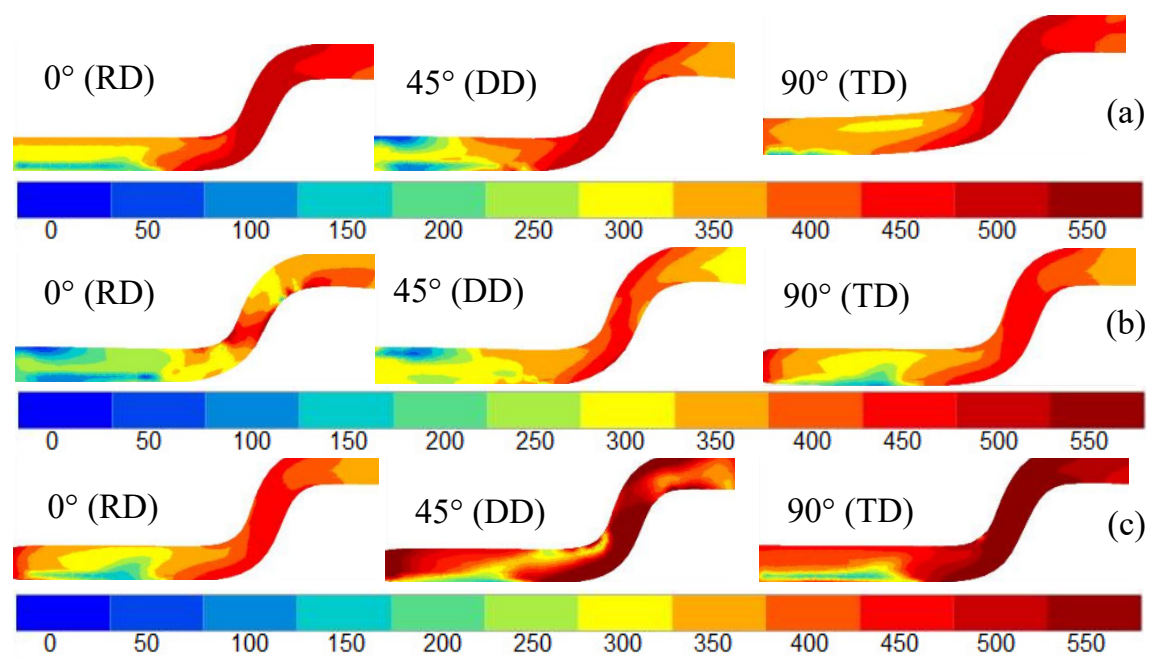

Figure 17: Contour plot of the equivalent stress at $8 \mathrm{~mm}$ depth: (a) unified model; (b) MM (2M1C) model; (c) polycrystalline model.

\section{Conclusion}

This paper presents an analysis of 2024-T351 aluminum alloy anisotropy using experimental tensile tests and three multi-scales approaches: Unified, MultiMechanisms and Polycrystalline. A good agreement is obtained between the experiments and the three proposed numerical models in terms of stress-strain behavior. It is shown that for the anisotropy simulation, the MM model and the polycrystalline one give more accurate results compared to the unified model. The major advantage of this type of models is the use of a reduced parameter's number. Starting from the above approaches and using model's identification techniques, numerical simulations of a simple drawing process has been analyzed. The loaddisplacement curves and the distribution of the equivalent stress show the feasibility of all proposed models. The next step of this work is to study a complete $3 \mathrm{D}$ anisotropy corresponding to large plastic deformations, starting from channel die compressions, a biaxial tensile test and simple shear ones. 


\section{References}

[1] Seidt, J.D., Plastic Deformation and Ductile Fracture of 2024-T351 Aluminum under Various Loading Conditions, Ph.D. Thesis, Graduate School of the Ohio State University, 2010.

[2] Ladevèze, P., Nonlinear Computational Structural Mechanics-New Approaches and Non-Incremental Methods of Calculation, Springer, 1999.

[3] Prager, W., The theory of plasticity - a survey of recent achievements. Proc. Inst. of Mech. Eng., London, England, 169, pp. 41-57, 1955.

[4] Voce, E., A practical strain hardening function, Metallurgia, 51 (307), pp. 219-226, 1955.

[5] Hill, E., A theory of the yielding and plastic flow of anisotropic metals, Proc. Roy. Soc., London, 1948.

[6] Zhang, S., Francillette, H., Gavrus, A., Analysis of the anisotropic behavior and the formability aptitude for an AA2024 alloy using channel die compression test and the simple tension test, Key Eng. Mat., 504-506, pp. 23-28, 2012.

[7] Cailletaud, G., Saï, K., A polycrystalline model for the description of ratchetting: Effect of intergranular and intragranular hardening, Mat. Sci. Eng., 480, pp. 24-39, 2008.

[8] Saï, K., Multi-mechanism models: Present state and future trends, Int. J. Plast, 27, pp. 250-281, 2011.

[9] Nasri, W., Gavrus, A., Kouadri, A., Saï, K., Applications of multi-scale models to numerical simulation and experimental analysis of anisotropic elastoplastic behavior of metallic sheets, Key Eng. Mat., 611-612, pp. 536544, 2014.

[10] Saï, K., Aubourg, V., Cailletaud, G., Strudel, J., Mater. Sci. Technol., 20, pp. 747-755, 2004. 